\title{
INFLUÊNCIA DE NITROGÊNIO E POTÁSSIO, VIA FERTIRRIGAÇÃO, EM ATRIBUTOS DO SOLO, NÍVEIS CRÍTICOS FOLIARES E PRODUÇÃO DO COQUEIRO-ANÃO(1)
}

\author{
Lafayette Franco Sobral $^{(2)} \&$ Luis Carlos Nogueira ${ }^{(2)}$
}

\begin{abstract}
RESUMO
O plantio de coqueiro-anão irrigado, com o objetivo de produzir coco para aproveitamento de água, tem se expandido muito no Brasil. As maiores produtividades, obtidas sob irrigação, influenciam nas quantidades de $\mathrm{N}$ e de K requeridas pela planta, o que altera as relações entre os seus teores no solo e na planta. Um experimento com plantas de coqueiro-anão, irrigado por microaspersão, foi conduzido em um Argissolo Amarelo da unidade de paisagem dos tabuleiros costeiros no Nordeste do Brasil. Foi utilizada a matriz Pan Puebla III em blocos ao acaso, com quatro repetições. Os tratamentos de fertirrigação foram dez combinações de $\mathrm{N}$ e $\mathrm{K}$, utilizando uréia e cloreto de potássio como fontes dos nutrientes, aplicados semanalmente durante 52 meses. Amostras de solo foram coletadas na zona de influência do microaspersor e amostras de folhas foram coletadas nas posições 9 e 14. A aplicação de uréia reduziu o $\mathrm{pH}$ e os teores de $\mathrm{Ca}^{2+}$ e $\mathrm{Mg}^{2+}$ no solo. O maior valor de condutividade elétrica do extrato de saturação do solo foi de $0,65 \mathrm{dS} \mathrm{m}^{-1}$, na profundidade $0-5 \mathrm{~cm}$, e decresceu com a profundidade. Os coeficientes de correlação entre os teores de $\mathrm{K}$ na folha e no solo, nas profundidades 0-5, 0-20 e 20-40 cm, foram similares, indicando que a amostragem de solo para fertilidade na cultura do coqueiro-anão fertirrigado pode ser feita na profundidade de 0-20 $\mathrm{cm}$. Maior quantidade de massa do fruto e mais volume do albúmen líquido foram obtidos com a menor dose de $\mathrm{N}$, dado o menor número de frutos por árvore. Os tratamentos de $\mathrm{N}$ e $\mathrm{K}$ não influenciaram o valor do pH da água do coco, porém alteraram o valor do brix. O teor de $\mathrm{K}$ na água do coco aumentou com a quantidade de $\mathrm{K}$ aplicada e do teor de $\mathrm{K}$ na folha. Os níveis críticos de $\mathrm{N}$ e $\mathrm{K}$ nas folhas 9 e 14 foram 19,2, 20,5, 12,1 e 9,4 $\mathrm{g} \mathrm{kg}^{-1}$, respectivamente. O nível crítico de K no solo pelo Mehlich-1, na profundidade 0-20 cm, foi $45 \mathrm{mg} \mathrm{dm}^{-3}$.
\end{abstract}

Termos de indexação: coqueiro, adubação, análise foliar.

\footnotetext{
(1) Recebido para publicação em agosto de 2006 e aprovado em maio de 2008.

(2) Pesquisadores da Embrapa Tabuleiros Costeiros. Avenida Beira Mar 3250, CEP 49025-040 Aracaju (SE). E-mails: lafayete@cpatc.embrapa.br; nogueira@cpatc.embrapa.br
} 


\title{
SUMMARY: INFLUENCE OF NAND K APPLIED BY FERTIRRIGATION ON SOIL PROPERTIES, CRITICAL LEAF LEVELS AND YIELD OF DWARF COCONUT
}

\begin{abstract}
Irrigated plantations of dwarf coconut for the production of coconut water have expanded considerably in Brazil. Higher yields obtained under irrigation affect the amount of $N$ and $K$ required by dwarf coconut, influencing the relationships between yield and soil and leaf contents of these nutrients. An experiment was set up on an Ultissol in the Brazilian Northeast coastal tablelands, following a Pan Puebla III experimental matrix in a randomized block design. Ten combinations of urea and potassium chloride were weekly injected during 52 months. Soil samples were collected in the irrigated area and leaf samples taken from the leaves 9 and 14. Soil $\mathrm{pH}$, Ca and $\mathrm{Mg}$ contents decreased as urea doses increased. The highest electrical conductivity value was $0.65 \mathrm{dS} \mathrm{m} \mathrm{m}^{-1}$ in the $0-5 \mathrm{~cm}$ layer and it decreased with depth. Correlation coefficients between leaf $K$ and soil $K$ in the layers $0-5,0-20$ and $20-40 \mathrm{~cm}$ were very similar, indicating that layer $0-20 \mathrm{~cm}$ can be sampled for fertility purposes in irrigated coconut. Highest fruit weight and coconut water volume were obtained with the lowest $N$ level, due to the lower number of nuts per tree. Nitrogen and K combinations did not influence the coconut water $\mathrm{pH}$, but increased coconut water brix. The $K$ content in the coconut water increased along the $K$ doses; leaf $K$ and $K$ in the coconut water were directly related. Nitrogen and $K$ critical levels for leaves 9 and 14 were 19.2 and $20.5 \mathrm{~g} \mathrm{~kg}^{-1}$ and 12.1 and $9.4 \mathrm{~g} \mathrm{~kg}^{-1}$, respectively. The critical level of soil $\mathrm{K}$ by Mehlich-1 in the $0-20 \mathrm{~cm}$ layer was $45 \mathrm{mg} \mathrm{dm}^{-3}$.
\end{abstract}

Index terms: coconut, fertilization, leaf analysis.

\section{INTRODUÇÃO}

Nos últimos 10 anos, tem sido observada grande expansão de plantios de coqueiros-anões irrigados, com produtividade superior à obtida em cultivos sem irrigação. Cuenca (1998) estima produtividade de 90 e 150 frutos planta ${ }^{-1}$ ano $^{-1}$ para o coqueiro-anão aos oito anos, sem e com irrigação, respectivamente.

As deficiências de $\mathrm{N}$ e K são as mais comuns nos coqueirais (Sobral, 1998), e as quantidades dos dois nutrientes removidas pelo coqueiro é alta, pois a planta desenvolve-se continuamente. Silva et al. (2005) observaram que, em área não irrigada, a remoção de $\mathrm{N}$ e K pelos frutos colhidos aos sete meses foi $23 \mathrm{e}$ $76 \mathrm{~kg} \mathrm{ha}^{-1} \mathrm{ano}^{-1}$, respectivamente. Os mesmos autores concluíram que, em área irrigada, a remoção foi de 74 e $243 \mathrm{~kg} \mathrm{ha}^{-1}$ ano $^{-1}$.

Sob fertirrigação, maiores requerimentos podem influenciar os níveis críticos foliares dos dois nutrientes. Holanda et al.,(2007) observaram que em coqueiro-anão verde fertirrigado, os níveis críticos de $\mathrm{N}$ e K na folha 14 estão entre 18,7 e $19,3 \mathrm{~g} \mathrm{~kg}^{-1}$ e entre 9 e $10 \mathrm{~g} \mathrm{~kg}^{-1}$, respectivamente. O nível critico de $\mathrm{K}$ é maior que a faixa de 6 a $8 \mathrm{~g} \mathrm{~kg}^{-1}$ proposta por Magat (2005) para a folha 14 do coqueiro-anão sem irrigação.

$\mathrm{O} \mathrm{N}$ e o K influenciam a qualidade dos frutos do coqueiro-anão. Teixeira et al. (2005a) concluíram que o $\mathrm{N}$ diminuiu o volume da água do coco e a massa do fruto, e o K teve efeito inverso. Silva et al. (2006) e Ferreira Neto et al. (2007) observaram que o N diminuiu o teor de sólidos solúveis e o $\mathrm{K}$ aumentou.
A aplicação freqüente de pequenas quantidades de $\mathrm{N}$ e K mantendo os nutrientes ao alcance das raízes é uma das vantagens da fertirrigação. Entretanto, a aplicação de fertilizantes pode influenciar os atributos químicos do solo, principalmente naqueles com baixos teores de argila e matéria orgânica. Teixeira et al. (2005b) concluíram que doses superiores a $240 \mathrm{~kg} \mathrm{ha}^{-1}$ de $\mathrm{N}$ na forma de nitrato de amônio provocaram acidificação do solo, diminuindo a saturação por bases. O comportamento produtivo do coqueiro em resposta à salinidade no solo foi avaliado em pesquisas no Rio Grande do Norte e na Paraíba (Ferreira Neto et al., 2002; Marinho et al., 2006).

Os objetivos deste trabalho foram: (a) estudar a influência do $\mathrm{N}$ e do K na produção e na qualidade dos frutos do coqueiro-anão fertirrigado; (b) determinar as relações entre teores de $\mathrm{K}$ no solo e de $\mathrm{N}$ e K na folha com a produção, visando à obtenção de níveis críticos no solo e na planta; e (c) estudar as modificações nos atributos químicos do solo, provocadas pela aplicação continuada de uréia e cloreto de potássio via água de irrigação.

\section{MATERIAL E MÉTODOS}

O experimento foi conduzido no Distrito de Irrigação Platô de Neópolis, cujas coordenadas geográficas são $10^{\circ} 17^{\prime} \mathrm{S} \mathrm{e} 36^{\circ} 38^{\prime} \mathrm{O}$, e altitude de 120 m. Na classificação de Köppen, o clima da região do baixo São Francisco, onde está localizado o Distrito, é do tipo semi-úmido, com temperatura e umidade médias de $26^{\circ} \mathrm{C}$ e $77 \%$ respectivamente. 
O solo da área do experimento foi classificado como Argissolo Amarelo, e nas profundidades 0-20 e 20$40 \mathrm{~cm}$, os teores de areia foram 892,1 e $863,3 \mathrm{~g} \mathrm{~kg}^{-1}$, os de silte 50,2 e $51,4 \mathrm{~g} \mathrm{~kg}^{-1}$ e os de argila $57,7 \mathrm{e}$ $85,2 \mathrm{~g} \mathrm{~kg}^{-1}$, respectivamente (Peixoto, 2001). Antes da aplicação dos tratamentos, amostras de solo foram coletadas por bloco nas profundidades 0-20 e $20-40 \mathrm{~cm}$. Os valores de $\mathrm{pH}$ foram 5,4 e 5,0, e os teores de matéria orgânica, K, $\mathrm{Na}, \mathrm{Ca}^{2+}$ e $\mathrm{Mg}^{+}$foram 15,1 e $12,6 \mathrm{~g} \mathrm{dm}^{-3}$, $13,6 \mathrm{mg} \mathrm{dm}^{-3}$ (nas duas profundidades), 7,4 e $6,0 \mathrm{mg} \mathrm{dm}^{-3}, 6,60$ e $3,3 \mathrm{mmol}_{\mathrm{c}} \mathrm{dm}^{-3}$ e 4,2 e $3,3 \mathrm{mmol}_{\mathrm{c}} \mathrm{dm}^{-3}$, respectivamente. $\mathrm{O}$ teor de $\mathrm{P}$ na profundidade $0-20 \mathrm{~cm}$ foi $8,3 \mathrm{mg} \mathrm{dm}^{-3}$ e os valores de CTC foram 33,0 e 30,2 $\mathrm{mmol}_{\mathrm{c}} \mathrm{dm}^{-3}$ nas duas profundidades.

$\mathrm{O}$ experimento foi instalado em um plantio de coqueiro-anão verde com quatro anos de idade, plantado em espaçamento $7,5 \times 7,5 \times 7,5 \mathrm{~m}$ em triângulo. A parcela útil foi constituída de seis plantas, separadas por uma linha neutra. A área do experimento foi de 2,86 ha. Foi utilizada a matriz Pan Puebla III modificada $\left(2^{\mathrm{k}}+2 \mathrm{xk}+1+1\right)$ em blocos ao acaso, com quatro repetições. Os tratamentos de fertirrigação foram dez combinações de $\mathrm{N}$ e K, cujas doses foram $135,810,1.350,1.890$ e 2.565 g planta $^{-1}$ ano $^{-1}$. Foram utilizadas as fontes uréia e cloreto de potássio, as quais foram aplicadas semanalmente, durante 52 meses. Foram feitas adubações em todos os tratamentos com $\mathrm{P}$ na forma de superfosfato triplo na dose de $1 \mathrm{~kg}$ planta ${ }^{-1}$; com $\mathrm{Mg}$ na forma de óxido de $\mathrm{Mg}$ na dose de $0,800 \mathrm{~kg}_{\text {planta-1; }}$; com $\mathrm{S}$ na forma de $\mathrm{S}$ elementar na dose $0,100 \mathrm{~kg}_{\text {planta }}{ }^{-1} ;$ com $\mathrm{B}$ na forma de ácido bórico na dose de $0,050 \mathrm{~kg}_{\text {planta }}{ }^{-1}$; com Zn na forma de sulfato de Zn na dose de $0,100 \mathrm{~kg}$ planta $^{-1}$ e com Mn na forma de sulfato de $\mathrm{Mn}$ na dose de $0,100 \mathrm{~kg}$ planta $^{-1}$. O Ca foi provido por meio da aplicação de calcário de forma localizada e a lanço, nas doses de 2,5 kg planta-1 e de $2 \mathrm{t} \mathrm{ha}^{-1}$.

$\mathrm{O}$ sistema de irrigação foi o de microaspersão, com dois emissores autocompensantes por planta, com vazão de $40 \mathrm{~L} \mathrm{~h}^{-1}$ cada, operando a uma pressão de serviço de $240 \mathrm{kPa}$. O monitoramento da pressão foi feito por manômetros instalados no início da tubulação dos tratamentos, e o volume de água aplicado diariamente foi de $150 \mathrm{~L}$ por planta.

Durante a condução do experimento, foram coletados dados de frutos por planta, consolidados para cada ano. Amostras dos frutos foram tomadas por tratamento, nas quais foram determinados a massa, o volume de água, o $\mathrm{pH}$ e o brix da água do coco. Amostras das folhas 9 e 14 foram coletadas anualmente para determinação do $\mathrm{N}$ e do K (Silva, 1999). Os teores de $\mathrm{P}, \mathrm{Ca}, \mathrm{Mg}, \mathrm{Mn}, \mathrm{Zn}, \mathrm{Cu}, \mathrm{Fe}, \mathrm{B}$ е o Cl também foram determinados para acompanhamento do estado nutricional das plantas.

Amostras de solo foram coletadas na área de influência do microaspersor, nas profundidades $0-5$ e 0-20 e 20-40 cm nas quais foram determinados o $\mathrm{pH}$ $\left(\mathrm{H}_{2} \mathrm{O}\right)$, o $\mathrm{K}$ e o Na pelo Mehlich-1 (modificado para uma relação solo: solução 1:10), o $\mathrm{Ca}^{2+}$ e o $\mathrm{Mg}^{2+}$ por meio de extração com $\mathrm{KCl} 1 \mathrm{~mol} \mathrm{~L}^{-1}$. Um outro conjunto de amostras de solo foi coletado nas profundidades $0-5,5-10,10-20$ e $20-40 \mathrm{~cm}$, para a determinação da condutividade elétrica do extrato de saturação, de acordo com Silva (1999).

Os dados médios de colheita das plantas com seis anos de idade foram submetidos às análises de variância e regressão utilizando-se o Sistema de Análise Estatística (SAS, 1994). Para o cálculo das doses econômicas, utilizou-se o método proposto por Black (1992). O número de frutos foi multiplicado por 0,20 (assumindo-se $\mathrm{R} \$ 0,20$ por fruto) e uma nova equação foi ajustada. A segunda derivada desta equação foi igualada à derivada do custo do fertilizante $\left(\mathrm{R} \$ 1,78 \mathrm{~kg}^{-1}\right.$ de $\mathrm{N}$ na uréia e $\mathrm{R} \$ 1,40 \mathrm{~kg}^{-1}$ de $\mathrm{K}$ no cloreto de K).

\section{RESULTADOS E DISCUSSÃO}

A aplicação da uréia via água de irrigação, durante 52 meses, causou decréscimo significativo no $\mathrm{pH}$ e nos teores de $\mathrm{Ca}^{2+} \mathrm{e} \mathrm{Mg}^{2+}$ no solo (Figura 1a,b,c) e ao longo do tempo, a aplicação de $2.565 \mathrm{~g} \mathrm{~N}$ na forma de uréia acentuou a acidificação do solo (Figura 1d). Esse efeito pode ser atribuído às reações de hidrólise da uréia no solo, que produzem $\mathrm{H}^{+}$e contribuem para decrescer o $\mathrm{pH}$ e os teores de $\mathrm{Ca}^{2+}$ e de $\mathrm{Mg}^{2+}$. Silva et al. (2005b) também observaram diminuição do $\mathrm{pH}$ e do teor de $\mathrm{Ca}^{2+}$ do solo com a fertirrigação com uréia. Teixeira et al. (2005b) observaram decréscimo do $\mathrm{pH}$ do solo e na saturação por bases, quando as doses de $\mathrm{N}$ foram maiores que $240 \mathrm{~kg} \mathrm{ha}^{-1}$ na forma de nitrato de amônio.

Os tratamentos não influenciaram significativamente a condutividade elétrica do extrato de saturação do solo (CEes) nas profundidades amostradas (Quadro 1). Depois de 52 meses de aplicações semanais de uréia e cloreto de potássio, o maior valor de CEes encontrado foi $0,65 \mathrm{dS} \mathrm{m}{ }^{-1}$ observado na profundidade $0-5 \mathrm{~cm}$, que decresceu com a profundidade. Ferreira Neto et al. (2002) aplicaram água com salinidade de até $15 \mathrm{dS} \mathrm{m} \mathrm{m}^{-1}$ e observaram que a CEes ficou acima de $4 \mathrm{dS} \mathrm{m}^{-1}$, sem, contudo, comprometer a produtividade do coqueiro. Entretanto, Marinho et al. (2006) observaram diminuição do peso e do número de frutos colhidos, quando a condutividade elétrica da água de irrigação foi maior que $5 \mathrm{dS} \mathrm{m} \mathrm{m}^{-1}$.

As relações entre o $\mathrm{N}$ aplicado via água de irrigação, a produção de frutos e os teores de $\mathrm{N}$ nas folhas 9 e 14 são mostradas na figura 2a,b,c. A dose de $\mathrm{N}$ que proporcionou a produção máxima foi $1.716 \mathrm{~g}$ de $\mathrm{N}$ planta ${ }^{-1}$ ano $^{-1}$, e a dose econômica foi $1.567 \mathrm{~g}$ de $\mathrm{N}_{\text {planta }}{ }^{-1}$ ano $^{-1}$ (Figura 2a). Os níveis críticos de $\mathrm{N}$ nas folhas 9 e 14, foram 19,2 e $20,5 \mathrm{~g} \mathrm{~kg}^{-1}$, respectivamente, obtidos relacionando-se os teores foliares com a produção de frutos (Figura 2b,c). O 
(a)
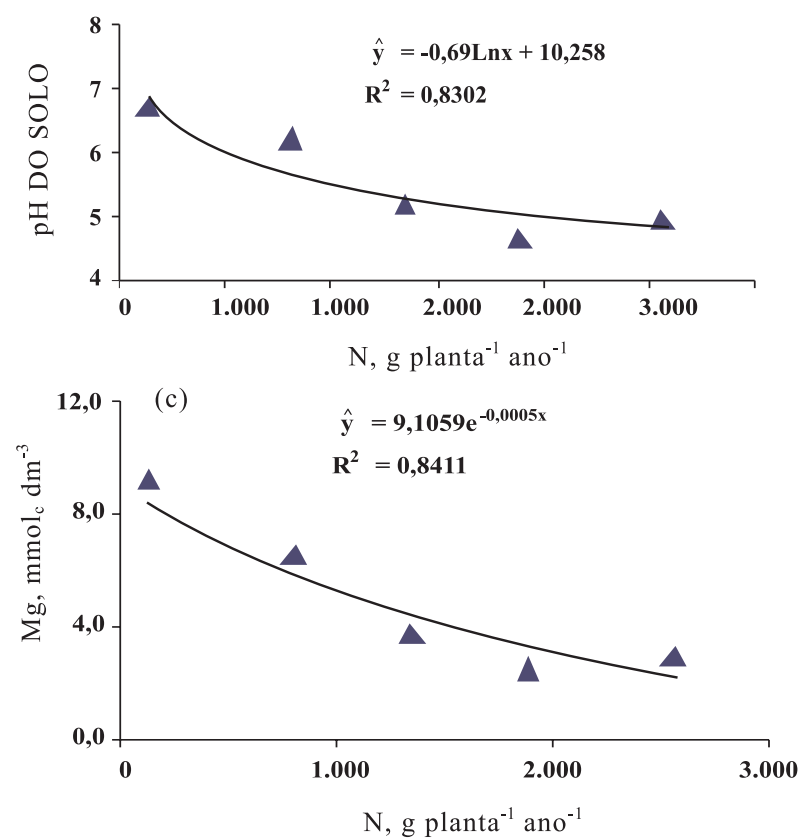

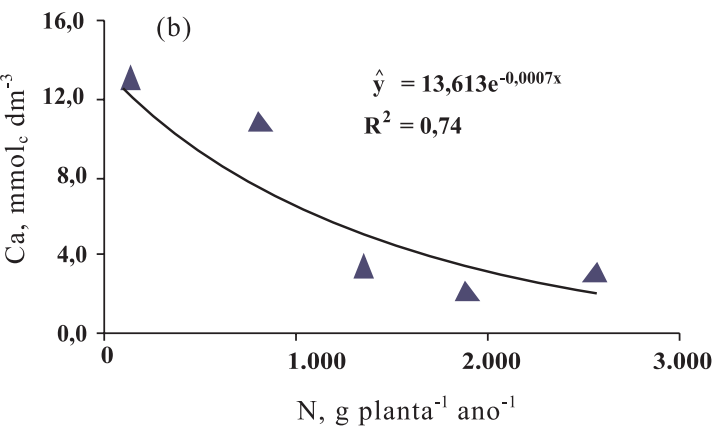

(d)

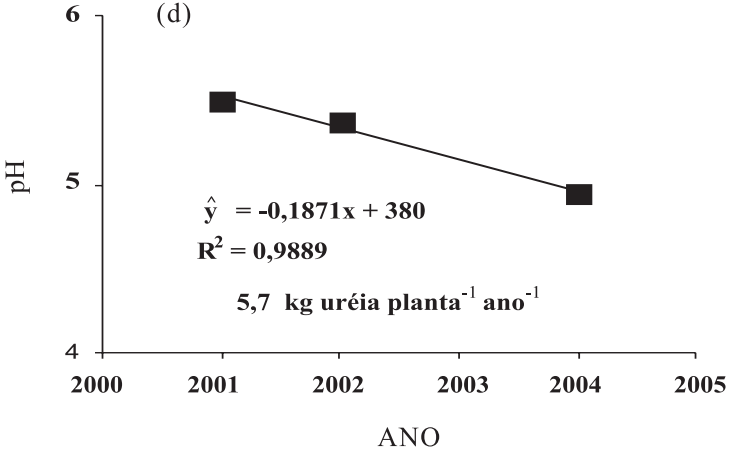

Figura 1. Influência de doses de $\mathrm{N}$-uréia no pH (a), nos teores de cálcio (b) e de magnésio trocáveis (c) e no decréscimo do pH em função do tempo (d) na profundidade 0-20 cm.

Quadro 1. Condutividade elétrica no extrato saturado de amostras coletadas em quatro profundidades do solo, na área de influência do microaspersor

\begin{tabular}{|c|c|c|c|c|c|}
\hline \multicolumn{2}{|c|}{ Tratamento } & \multicolumn{4}{|c|}{ Profundidade } \\
\hline $\mathbf{N}$ & $\mathbf{K}$ & $0-5 \mathrm{~cm}$ & $5-10 \mathrm{~cm}$ & $10-20 \mathrm{~cm}$ & $20-40 \mathrm{~cm}$ \\
\hline \multicolumn{2}{|c|}{ - g planta ${ }^{-1}$ ano $^{-1}$} & & 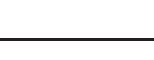 & & 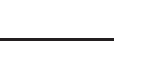 \\
\hline 810 & 810 & 0,31 & 0,44 & 0,29 & 0,28 \\
\hline 810 & 1.890 & 0,62 & 0,53 & 0,31 & 0,28 \\
\hline 1.890 & 810 & 0,41 & 0,31 & 0,34 & 0,25 \\
\hline 1.890 & 1.890 & 0,59 & 0,34 & 0,36 & 0,40 \\
\hline 135 & 810 & 0,65 & 0,40 & 0,37 & 0,40 \\
\hline 2.565 & 1.890 & 0,37 & 0,31 & 0,32 & 0,31 \\
\hline 810 & 135 & 0,53 & 0,32 & 0,27 & 0,34 \\
\hline 1.890 & 2.565 & 0,52 & 0,40 & 0,37 & 0,38 \\
\hline 1.350 & 1.350 & 0,41 & 0,42 & 0,31 & 0,25 \\
\hline 135 & 135 & 0,42 & 0,31 & 0,27 & 0,31 \\
\hline \multicolumn{2}{|c|}{$\mathrm{F}$} & $1,84 \mathrm{~ns}$ & $1,16 \mathrm{~ns}$ & $1,08 \mathrm{~ns}$ & $1,00 \mathrm{~ns}$ \\
\hline \multicolumn{2}{|c|}{ CV (\%) } & 35,32 & 36,07 & 23,26 & 35,49 \\
\hline
\end{tabular}

nível crítico $\mathrm{N}$ na folha 14 foi ligeiramente superior à faixa de 18 a $20 \mathrm{~g} \mathrm{~kg}^{-1}$ proposta Magat (2005) para o coqueiro-anão sem irrigação.

As relações entre o $\mathrm{K}$ aplicado via água de irrigação, a produção de frutos, e os teores de K nas folhas 9 e 14 são mostradas na figura 3. A dose de $\mathrm{K}_{2} \mathrm{O}$ que maximizou a produção de frutos foi $1.892 \mathrm{~g}$ planta $^{-1}$ ano $^{-1}$, e a dose econômica foi $1.654 \mathrm{~g}$ de $\mathrm{K}_{2} \mathrm{O}$ planta $^{-1}$ ano $^{-1}$ (Figura 3a). Os níveis críticos de $\mathrm{K}$ nas folhas 9 e 14, calculados a partir das equações da figura 3b,c, foram 12,1 e 9,4 $\mathrm{g} \mathrm{kg}^{-1}$, respectivamente, sendo ambos superiores à faixa de 6 a $8 \mathrm{~g} \mathrm{~kg}^{-1}$ proposta por Magat (2005) para a folha 14 do coqueiro-anão sem irrigação.

As diferenças entre níveis críticos de coqueiros-anões irrigados e não irrigados foram maiores para o K, pois este nutriente é removido pelos frutos em maior quantidade que o N (Silva et al., 2005). Esses resultados 

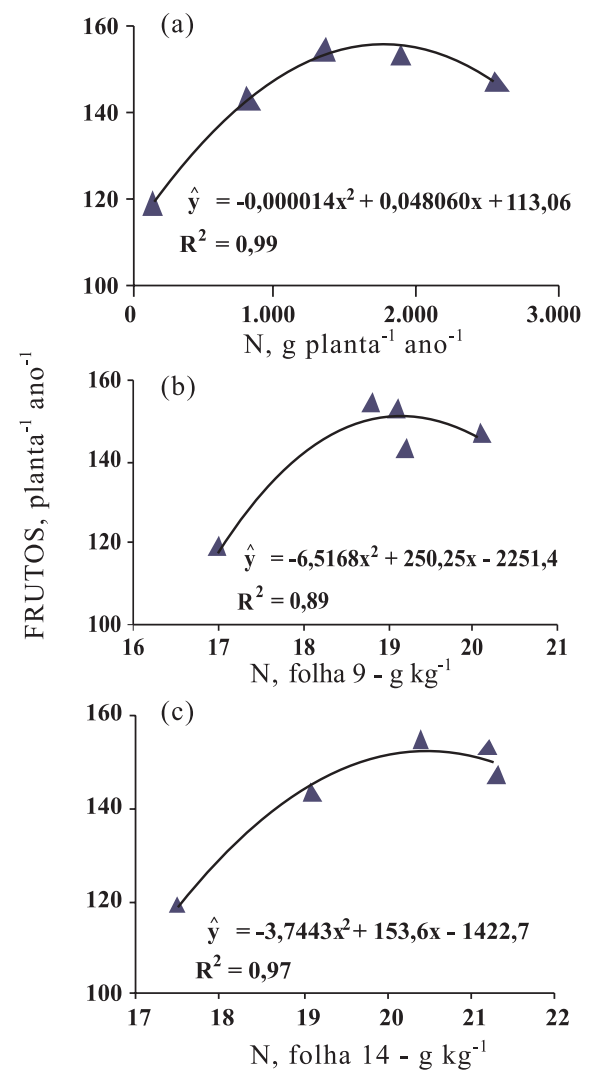

Figura 2. Influência do $\mathrm{N}$ na produção de frutos(a) e relação entre o teor foliar de $\mathrm{N}$ nas folhas 9 (b) e 14 (c) e a produção de frutos em coqueiros de seis anos de idade. confirmam a hipótese de que, sob irrigação, o alto potencial de produtividade e o contínuo suprimento de água e de nutrientes, aplicados via fertirrigação, alteram as relações entre os teores na folha.

Os teores de K no solo pelo método Mehlich-1, nas profundidades 0-5, 0-20 e 20-40 cm, foram relacionados aos teores de $\mathrm{K}$ nas folhas 9 e 14 . Os coeficientes de determinação para a folha 9 foram 0,88, 0,86 e 0,87 , e para a folha 14 foram $0,89,0,89$ e 0,86 , os quais são muito próximos. Estes resultados indicam que a amostragem feita na área de influência do microaspersor, na profundidade 0-20 cm atualmente estudada, é adequada para estimar o teor de $\mathrm{K}$ no solo. O nível crítico de K no solo pelo Mehlich-1, para a profundidade $0-20 \mathrm{~cm}$, foi estimado em $45 \mathrm{mg} \mathrm{dm}^{-3}$ (Figura 3d).

A análise de variância mostrou efeitos significativos dos tratamentos nas variáveis massa do fruto, volume do albúmen líquido e brix da água do coco. Não foram observados efeitos significativos no $\mathrm{pH}$ da água do coco (Quadro 2). A massa do fruto variou de 2.655,2 a $3.078,2 \mathrm{~g}$, valor superior ao obtido por Silva et al. (2006): 2,23 kg. Doses maiores de N causaram diminuição do tamanho do fruto dado o maior número de frutos produzidos (Figura 4a,b). O volume do albúmen líquido variou de 550,8 a 612,5 mL, maior que o observado por Silva et al. (2006): $417 \mathrm{~mL}$, e que o observado por Ferreira Neto et al. (2207): $500 \mathrm{~mL}$, com a menor dose de N. A variação do brix foi de 5,30 a 5,80, valor menor que o encontrado por Teixeira et al. (2005a): 7,0 a 7,5. Esses valores estão associados

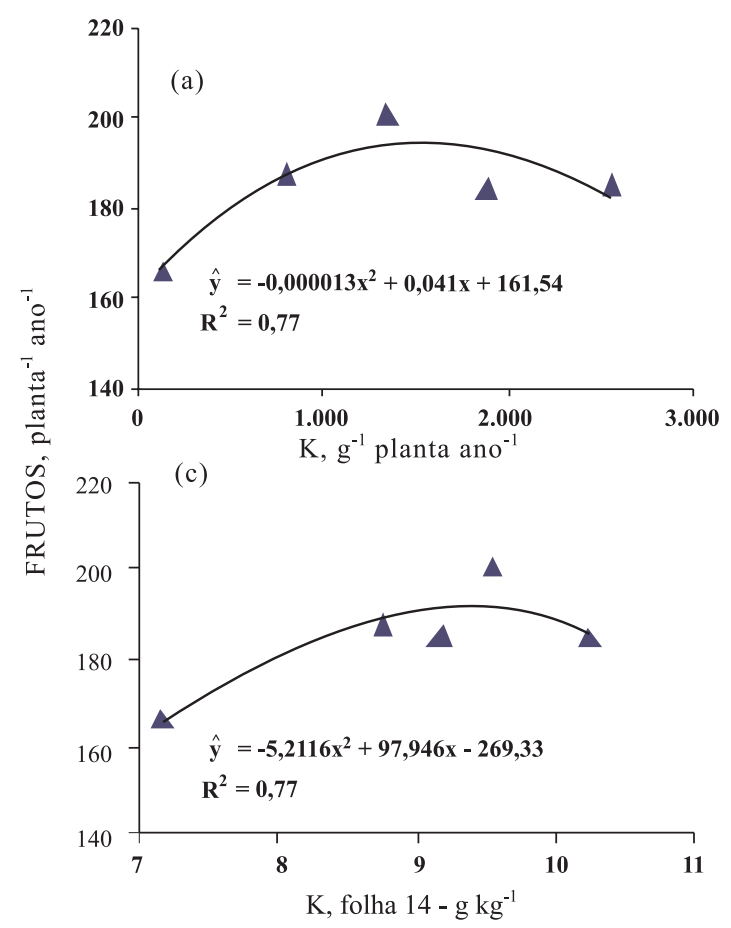

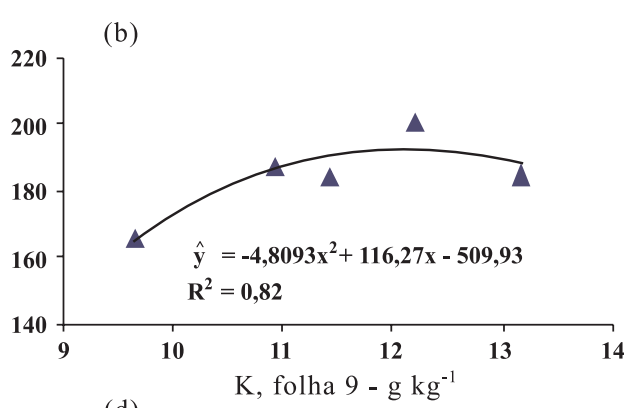

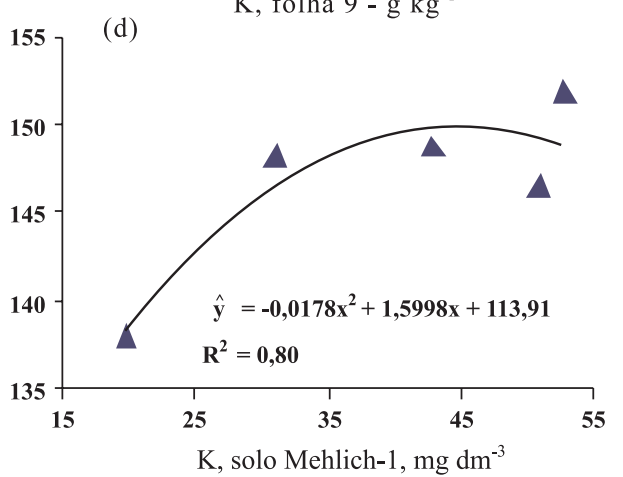

Figura 3. Efeitos do K na produção de frutos (a) e relação entre os teores de K nas folhas 9 (b), 14 (c), no solo(d), e a produção de frutos (d) em coqueiros de seis anos de idade. 
Quadro 2. Peso de fruto, volume de albúmen líquido, brix e pH da água de coco, em função de doses de $\mathrm{N}$ e K aplicados via água de irrigação

\begin{tabular}{|c|c|c|c|c|c|}
\hline \multicolumn{2}{|c|}{ Tratamento } & \multirow{2}{*}{ Massa do fruto } & \multirow{2}{*}{ Volume de albúmen líquido } & \multicolumn{2}{|c|}{ Água do coco colhido aos seis meses } \\
\hline \multicolumn{2}{|c|}{ N e K } & & & Brix & $\mathrm{pH}$ \\
\hline \multicolumn{2}{|c|}{ - g planta $^{-1}$ ano $^{-1}-$} & $\mathrm{g}$ & $\mathrm{mL}$ & & \\
\hline 810 & 810 & $2828,0 \mathrm{ab}$ & $563,7 \mathrm{ab}$ & $5,30 \mathrm{~b}$ & 5,02 a \\
\hline 1.890 & 1.890 & $2887,7 \mathrm{ab}$ & $598,3 \mathrm{ab}$ & $5,62 \mathrm{ab}$ & 5,12 a \\
\hline 1.890 & 810 & $2765,3 \mathrm{ab}$ & $591,7 \mathrm{ab}$ & $5,57 \mathrm{ab}$ & $5,15 \mathrm{a}$ \\
\hline 135 & 1.890 & $2874,7 \mathrm{ab}$ & $587,1 \mathrm{ab}$ & $5,65 \mathrm{ab}$ & $5,10 \mathrm{a}$ \\
\hline 2.565 & 810 & $3078,2 \mathrm{a}$ & $685,3 \mathrm{a}$ & $5,52 \mathrm{ab}$ & $5,17 \mathrm{a}$ \\
\hline 810 & 1.890 & $2831,7 \mathrm{ab}$ & $594,6 \mathrm{ab}$ & $5,80 \mathrm{a}$ & $5,07 \mathrm{a}$ \\
\hline 1.890 & 135 & $2875,9 \mathrm{ab}$ & $612,5 \mathrm{ab}$ & $5,47 \mathrm{ab}$ & $5,10 \mathrm{a}$ \\
\hline 1.890 & 2.565 & $2673,0 \mathrm{~b}$ & $587,4 \mathrm{ab}$ & $5,67 \mathrm{ab}$ & 5,12 a \\
\hline 1.350 & 1.350 & $2655,2 \mathrm{~b}$ & $550,8 \mathrm{~b}$ & $5,72 \mathrm{a}$ & $5,15 \mathrm{a}$ \\
\hline 135 & 135 & $2959,7 \mathrm{ab}$ & $653,3 \mathrm{ab}$ & $5,52 \mathrm{ab}$ & 5,12 a \\
\hline \multicolumn{2}{|c|}{$\mathrm{F}$} & $2,58^{*}$ & $2,41^{*}$ & $3,01^{*}$ & $0,72 \mathrm{~ns}$ \\
\hline \multicolumn{2}{|c|}{ CV (\%) } & 5,53 & 8,54 & 2,92 & 1,97 \\
\hline
\end{tabular}

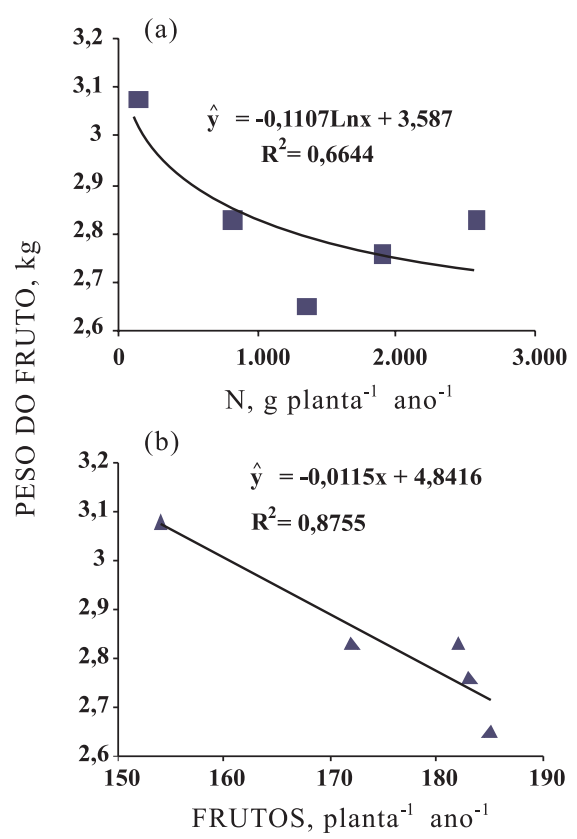

Figura 4. Massa do fruto de coqueiro-anão verde de seis anos de idade em função de doses de $\mathrm{N}$ (a) e do número de frutos por planta (b).

a valores de volume de água de coco bem menores que os encontrados neste trabalho, indicando um possível efeito diluição. Os valores de $\mathrm{pH}$ da água de coco estão próximos daqueles obtidos por Teixeira et al. (2005a): 4,9 a 5,2. As doses econômicas 1.716 e 1.654 g planta $^{-1}$ ano $^{-1}$ de $\mathrm{N}$ e $\mathrm{K}_{2} \mathrm{O}$ estão associadas a uma massa de fruto superior a $2.600 \mathrm{~g}$, a um volume de água superior a $550 \mathrm{~mL}$ e a um brix superior a 5,3. Em situações em que o volume de água é menor, pode-se obter um brix maior (Teixeira et al., 2005a).
Tanto o K aplicado quanto o teor de K da folha 14 influenciaram o teor de K na água de coco (Figura 5a,b). Teixeira et al. (2005a) sugerem que o teor de $\mathrm{K}$ pode estar associado à aceitabilidade da água de coco. Entretanto, os autores não observaram diferenças na aceitabilidade, quando as doses de $\mathrm{K}_{2} \mathrm{O}$ variaram de 0

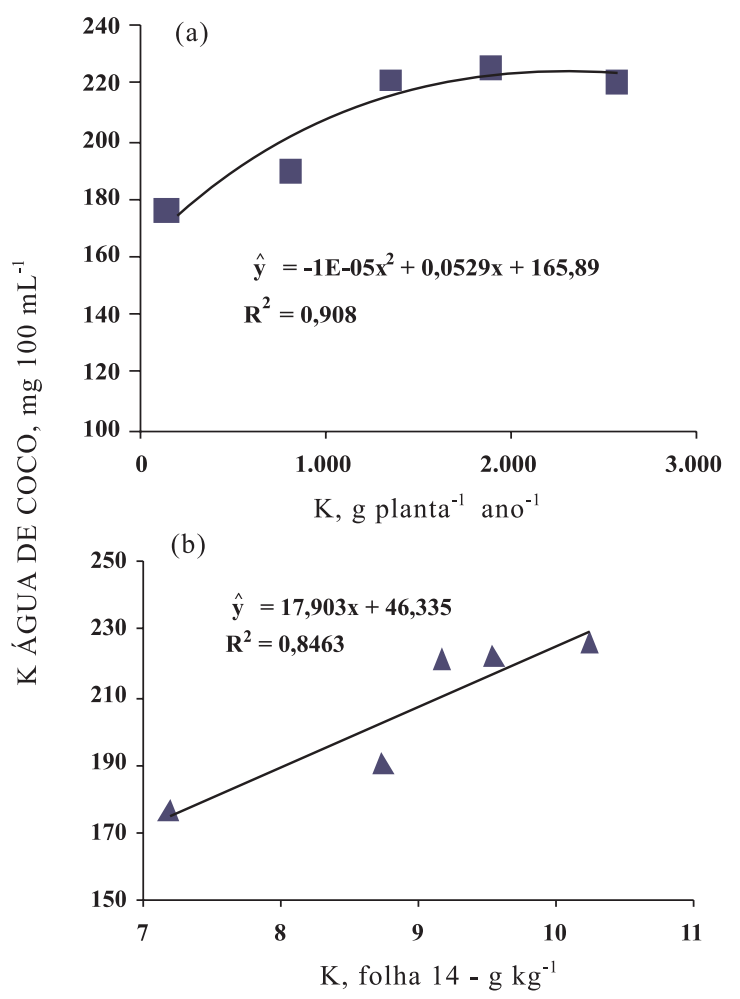

Figura 5. Teor de K na água do coco do coqueiro-anão verde em função do $K$ aplicado (a) e do teor de $K$ na folha $14(\mathrm{~b})$. 
a $360 \mathrm{~kg} \mathrm{ha}^{-1}$. Da figura $5 \mathrm{a}$, pode-se inferir que $172,84 \mathrm{mg} \mathrm{K} 100 \mathrm{~mL}^{-1}$ de água de coco corresponde a uma dose de $135 \mathrm{~g} \mathrm{~K}$ planta $^{-1} \mathrm{ano}^{-1}$ e que $235,78 \mathrm{mg}$ $\mathrm{K} 100 \mathrm{~mL}^{-1}$ de água de coco corresponde a uma dose de $2.565 \mathrm{~g} \mathrm{~K}$ planta $^{-1}$ ano $^{-1}$. A partir deste intervalo, pode-se criar uma tabela de recomendações de $\mathrm{K}$ com base na análise da água do coco. Entretanto, como o teor de K na água variou com o estádio de maturação, a época de amostragem é um fator crítico.

\section{CONCLUSÕES}

1. A aplicação de uréia reduziu o pH e os teores de $\mathrm{Ca}^{2+}$ e $\mathrm{Mg}^{2+}$ no solo.

2. A aplicação de uréia e $\mathrm{KCl}$ via fertirrigação, durante cinqüenta e dois meses, elevou a condutividade elétrica do extrato saturado para um valor máximo de $0,65 \mathrm{dS} \mathrm{m}^{-1}$.

3. A amostragem de solo para fertilidade na cultura do coqueiro-anão fertirrigado pode ser feita na profundidade de $0-20 \mathrm{~cm}$.

4. Os tratamentos não influenciaram o $\mathrm{pH}$ da água do coco, porém influenciaram o peso do fruto, o volume de água e o Brix da água do coco.

5. O teor de $\mathrm{K}$ na água do coco aumentou com as quantidades de $\mathrm{K}$ aplicadas e com o teor de $\mathrm{K}$ na folha.

6. Os níveis críticos de $\mathrm{N}$ e $\mathrm{K}$ nas folhas 9 e 14 foram 19,2 e 20,5 $\mathrm{g} \mathrm{kg}^{-1}$ e 12,1 e 9,4 $\mathrm{g} \mathrm{kg}^{-1}$, respectivamente.

7. O nível crítico de $\mathrm{K}$ no solo pelo Mehlich-1, na profundidade $0-20 \mathrm{~cm}$, foi $45 \mathrm{mg} \mathrm{dm}^{-3}$.

8. As doses que maximizaram a produção de coco foram $1.716 \mathrm{~g}$ de $\mathrm{N}$ e $1892 \mathrm{~g} \mathrm{de}_{2} \mathrm{O}$ planta $^{-1}$ ano $^{-1}$.

\section{AGRADECIMENTOS}

Ao Assistente de Pesquisa Erivaldo Fonseca Moraes e aos estagiários, representados por Derivan Santos da Silva (in memorian), pela assistência durante a condução do experimento. Aos proprietários e funcionários da Empresa H Dantas, nas pessoas do Sr. Marcelo Prado e do Sr. Hildeberto Barboza dos Santos, pela importante colaboração para este trabalho.

\section{LITERATURA CITADA}

CUENCA, M.A.G. Importância econômica do coqueiro. In: FERREIRA, J.M.S.; WARWICK, D.R.N. \& SIQUEIRA, L.A., eds. A cultura do coqueiro no Brasil. Aracaju, Embrapa, 1998. p.17-56.
BLACK, C.A. Soil fertility evaluation and control. Boca Raton, Lewis Publishers, 1992. p.79-144.

FERREIRA NETO, M.; GHEYI, H.R.; HOLANDA, J.S.; MEDEIROS, J.F. \& FERNANDES, P.D. Qualidade de fruto verde de coqueiro em função da irrigação com água salina. R. Bras. Eng. Agríc. Amb., 6:69-75, 2002.

FERREIRA NETO, M.; HOLANDA, J.S.; FOLEGATTI, M.V.; GHEYI, H.R.; PEREIRA, W.E. \& CAVALCANTE, L.F. Qualidade de fruto de coqueiro-anão verde em função de nitrogênio e potássio na fertirrigação. R. Bras. Eng. Agríc. Amb., 11:453-458, 2007.

HOLANDA, J.S.; FERREIRA NETO,M.; SILVA, R.A.; CHAGAS, M.C.M.; SOBRAL, L.F. \& GHEYI, H.R. Tecnologias para a produção intensiva de coco anão verde. Natal, EMPARN, 2007. (Boletim de Pesquisa, 34)

MAGAT, S.S. Coconut. In: World fertilizer use manual. International Fertilizer Association, 2005. Disponível em: $<$ http://www.fertilizer.org, Acesso em 04 Junho 2007.

MARINHO, J.L.M.; GHEYI, H.R.; FERNANDES, P.D.; HOLANDA, J.S. \& FERREIRA NETO, M. Cultivo do coco "Anão Verde" irrigado com águas salinas. Pesq. Agropec. Bras., 41:1277-1284, 2006.

PEIXOTO, J.F.S. Teores de macro e micronutrientes na cultura do coqueiro-anão verde fertirrigado com $\mathrm{N}$ e $\mathrm{K}$ no nordeste do Brasil. Campina Grande, Universidade Federal da Paraíba, 2001. (Tese de Mestrado)

SILVA, F.C., org. Manual de análises químicas de solos, plantas e fertilizantes. Brasília, Embrapa Comunicação para Transferência de Tecnologia, 1999. 370p.

SILVA, J.Q.; RAMOS, J.M.V.; REIS, H.S. \& LIMA, E. Exportação de nutrientes pelo fruto do coqueiro-anão verde em área irrigada no litoral sul do Rio de Janeiro. In: CONGRESSO BRASILEIRO DE CIÊNCIA DO SOLO, 30., Recife, 2005. Anais. Recife, Sociedade Brasileira de Ciência do Solo, 2005a. CD-ROM.

SILVA, R.A.; HOLANDA, J.S.; CAVALCANTE, L.F.; FERREIRA NETO, M.; GUERRA, A.G. \& MOURA, M.F. Teores de Al, P, Ca, Mg e pH em três camadas de um solo quartzarênico fertirrigado com nitrogênio e potássio e cultivado com coqueiro-anão verde. In: CONGRESSO BRASILEIRO DE CIÊNCIA DO SOLO, 30., Recife, 2005. Anais. Recife, Sociedade Brasileira de Ciência do Solo, 2005b. CD-ROM.

SILVA, R.A.; CAVALCANTE, L.F.; HOLANDA, J.S.; PEREIRA,W.E.; MOURA, M.F. \& FERREIRA NETO, M. Qualidade de frutos do coqueiro-anão verde fertirrigado com nitrogênio e potássio. R.. Bras. Frutic., 28:310-313, 2006.

SOBRAL, L.F. Nutrição e Adubação do coqueiro. In: FERREIRA, J.M.S.; WARWICK, D.R.N. \& SIQUEIRA, L.A., eds. A Cultura do coqueiro no Brasil. Aracaju, Embrapa, 1998. p.129-157.

SAS Institute. Statistical Analysis System. User's guide. Cary, 1994. 
TEIXEIRA, L.A.J.; BATAGLIA, O.C.; BUZZETI, S. \& FURLANI JUNIOR, E. Adubação com NPK em coqueiro-anão-verde (Cocos nucifera L.) - Rendimento e qualidade de frutos. R. Bras. Frutic., 27:120-123, 2005a.
TEIXEIRA, L.A.J.; BATAGLIA, O.C.; BUZZETI, S. \& FURLANI JUNIOR, E. Adubação com NPK em coqueiro-anão-verde (Cocos nucifera L.) - Atributos químicos do solo e nutrição da planta. R. Bras. Frutic., 27:115-119, 2005b. 